\title{
UFV-P2 as a member of the Luz24likevirus genus: a new overview on comparative functional genome analyses of the LUZ24-like phages
}

\author{
Monique R Eller ${ }^{1}$, Pedro M P Vidigal ${ }^{2}$, Rafael L Salgado ${ }^{3}$, Maura P Alves ${ }^{1}$, Roberto S Dias ${ }^{4}$, Cynthia C da Silva ${ }^{4,6}$,
} Antônio F de Carvalho ${ }^{1}$, Andrew Kropinski ${ }^{7,8}$ and Sérgio $O$ De Paula ${ }^{4,5^{*}}$

\begin{abstract}
Background: Phages infecting spoilage microorganisms have been considered as alternative biocontrol agents, and the study of their genomes is essential to their safe use in foods. UFV-P2 is a new Pseudomonas fluorescens-specific phage that has been tested for its ability to inhibit milk proteolysis.

Results: The genome of the phage UFV-P2 is composed of bidirectional modules and presented 75 functionally predict ORFs, forming clusters of early and late transcription. Further genomic comparisons of Pseudomonas-specific phages showed that these viruses could be classified according to conserved segments that appear be free from genome rearrangements, called locally collinear blocks (LCBs). In addition, the genome organization of the phage UFV-P2 was shown to be similar to that of phages PaP3 and LUZ24 which have recently been classified as a Luzz4likevirus. Conclusions: We have presented the functional annotation of UFV-P2, a new Pseudomonas fluorescens phage. Based on structural genomic comparison and phylogenetic clustering, we suggest the classification of UFV-P2 in the Luz24likevirus genus, and present a set of shared locally collinear blocks as the genomic signature for this genus.
\end{abstract}

\section{Background}

According to the International Committee of Virus Taxonomy (ICTV) classification scheme based on morphology, biological characteristics and genome organization (http://www.ictvonline.org/virusTaxonomy.asp), the bacteriophage family Podoviridae contains two subfamilies and 11 genera, and the Luz24likevirus genus comprises the Pseudomonas-infecting bacteriophages PaP3 [1] and LUZ24 [2]. Beyond PaP3 and LUZ24, the phages tf [3], MR299-2 [4], PaP4(KC294142), vB_PaeP_p2-10_Or1 (HF543949) and vB_PaeP_C1-14_Or (HE983844) have similar genomic compositions and should be classified to this genus.

Pseudomonas fluorescens bacteriophage UFV-P2 [5], is a virus with a high ability to reduce casein proteolysis in milk. Milk proteolysis is caused by thermo-resistant

\footnotetext{
* Correspondence: depaula@ufv.br

${ }^{4}$ Laboratory of Molecular Immunovirology, Federal University of Viçosa, Av. PH Rolfs, s/n, Campus da UFV, Viçosa, Minas Gerais 36570-000, Brazil ${ }^{5}$ Department of General Biology, Federal University of Viçosa, Av. PH Rolfs, s/n, Campus da UFV, Viçosa, Minas Gerais 36570-000, Brazil

Full list of author information is available at the end of the article
}

enzymes produced by psychrotrophs and is responsible for serious losses in the dairy industry due to negative effects on the quality and reduced shelf life of dairy products. In this environment, Pseudomonas spp. are prevalent contaminants [6-8], mainly $P$. fluorescens $[9,10]$. The use of phages in biocontrol has been suggested as an alternative to the use of chemicals. For example, P. fluorescens-specific phages had been studied to control Pseudomonas population and as sanitation agents to efficiently remove bacterial biofilms on stainless steel surfaces similar to those used in food industries, where these contaminants are common [11-13]. However, they must be used with caution. In addition to proteolysis reduction and biofilm inhibition studies and, their host range determination, it is necessary to understand phages' genome and proteome to make possible their use as biocontrol agents.

To expand our understanding about the P. fluorescensspecific phage UFV-P2, we present in detail the analysis of its structural genome and its comparisons to other phage genomes.

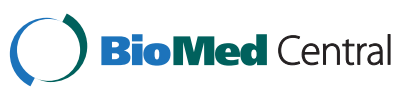

(c) 2014 Eller et al.; licensee BioMed Central Ltd. This is an open access article distributed under the terms of the Creative Commons Attribution License (http://creativecommons.org/licenses/by/2.0), which permits unrestricted use, distribution, and reproduction in any medium, provided the original work is properly cited. 


\section{Methods}

\section{Sampling}

The phage UFV-P2 was isolated from wastewater of a dairy industry in Minas Gerais, Brazil, and propagated at $30^{\circ} \mathrm{C}$ in $\mathrm{LB}$ medium in a strain of $P$. fluorescens $07 \mathrm{~A}$, courtesy of Laboratory of Food Microbiol, located at the Federal University of Viçosa, Brazil.

\section{Genome extraction and composition}

Phages were propagated in LB medium containing the bacteria in exponential phase. After incubation at $30^{\circ} \mathrm{C}$ for $8 \mathrm{~h}$, particle assemble was induced with mitomicin and the virions were recovered by centrifugation and filtration. Phage suspensions were incubated with $75 \mu \mathrm{g} / \mathrm{mL}$ of proteinase $\mathrm{K}$ in the presence of $0.01 \% \mathrm{SDS}$ at $56^{\circ} \mathrm{C}$ for $90 \mathrm{~min}$. Proteins were removed by extraction with phenol, phenol:chloroform (1:1), followed by chloroform. Genetic material was concentrated with an equal volume of isopropanol and resuspended in $30 \mu \mathrm{L}$ of distilled water. For analysis of viral genome composition, $5 \mu \mathrm{L}$ of the genomic extracts were submitted to digestion assays with enzymes DNase I $(50 \mu \mathrm{g} / \mathrm{mL})$ or RNaseA $(100 \mu \mathrm{g} / \mathrm{mL})$ for $60 \mathrm{~min}$ at $37^{\circ} \mathrm{C}$, followed by $1 \%$ agarose gel electrophoresis and visualization by staining with GelRed (Biotium, USA).

\section{Genomic DNA sequencing and assembly}

UFV-P2 genome was sequenced using an Illumina Genome Analyzer II by CD Genomics (New York, USA) and was assembled and analyzed using CLC Genomics Workbench version 5.1 (CLC bio, Cambridge, MA, USA). The sequence reads were assembled into contigs using stringent parameters, in which $90 \%$ of each read had to cover the other read with $90 \%$ identity. The data are available in GenBank database under accession number JX863101.

\section{Bioinformatics analysis}

The genome of phage UFV-P2 was oriented to be collinear with that of the type species, Pseudomonas phage LUZ24, and manually annotated using Kodon (Applied Maths, Austin, TX, USA.) [14]. The GenBank flat file (gbk) file was exported from Kodon and converted to FASTA-formatted protein sequences using gbk2faa (http:// lfz.corefacility.ca/gbk2faa/). The latter were screened for viral homologs using the BLASTP feature of Geneious R6.1 (Biomatters Ltd., Auckland, New Zealand); and, for protein motifs, using TMHMM [15], Phobius [16] and Batch Web CD-Search Tool [17] at http://www.ncbi.nlm. nih.gov/Structure/bwrpsb/bwrpsb.cgi.

Putative promoters were identified using the Kodon sequence similarity search feature employing TTGACA (N15-18)TATAAT and allowing for a 2 bp mismatch. Rho-independent terminators were tentatively identified using ARNold $[18,19]$ at http://rna.igmors.u-psud.fr/ toolbox/arnold/index.php.
For comparative purposes at the genomic level EMBOSS Stretcher [20] and progressive Mauve [21] were employed; while at the proteomic level we used CoreGenes [22,23]. Seventeen genomic reference sequences of phages were downloaded from GenBank (Table 1) and compared to UFV-P2 genome.

\section{Phylogenetic clustering}

For clustering UFV-P2 phage in an evolutionary way, a phylogenetic hypothesis was inferred by Bayesian inference (BI) using MrBayes v3.2.2 [24]. The genomic sequences of phages were aligned using ClustalW [25], and a pairwise distance matrix was calculated MEGA version 5 [26] (Table 1). The alignment was manually inspected, and the sites with gaps were excluded. To expedite the construction of phylogenetic trees, a model of nucleotide substitution was estimated using the jModelTest 2 program [27]. The GTR + G substitution model was selected as the best DNA evolution model for genomic sequences, according to the Akaike Information Criterion (AIC) and Bayesian Information Criterion (BIC).

The BI phylogenetic tree was calculated using the Bayesian Markov Chain Monte Carlo (MCMC) method, in two runs with 5,000,000 generations. The convergence of the parameters was analyzed in TRACER v1.5.0 (http://beast.bio.ed.ac.uk/tracer), and the chains reached a stationary distribution after 50,000 generations. Then, a total of $1 \%$ of the generated trees was burned to produce the consensus tree. To root the phylogenetic tree, the Enterobacteria phage T7 (NC_001604) was selected as outgroup taxa.

\section{Results and Discussion}

Transmission electron microscopy of the UFV-P2 virions (data not shown) showed that this virus has isometric capsids and very short tails, with morphological similarity to the $P$. aeruginosa phages Pap3 and MR299-2. Thus, UFV-P2 can be inserted in the Podoviridae family, order Caudovirales.

\section{Functional genomic organization \\ The viral genome was extracted and sequenced}

The phage UFV-P2 has a linear 45,517 bp DNA genome with a GC content of $51.5 \%$, and was sequenced with coverage of 30,655 fold. One of the interesting characteristics of members of the Luz24likevirus genus is the presence of localized single-stranded breaks associated with the consensus sequence TACTRTGMC [28]. Fourteen of these sequences were found in the top strand of the tf DNA, while the genome of UFV-P2 contains 15.

At first, bioinformatics analyses had showed that the UFV-P2 genome has a bidirectional organization with 92 predicted open reading frames (ORFs) larger than $100 \mathrm{bp}$, but only 41 ORFs (44.75\%) could be identified as 
Table 1 Pairwise comparisons of phage UFV-P2 and others phage genomes

\begin{tabular}{|c|c|c|c|c|c|}
\hline \multirow[b]{2}{*}{ Phage } & \multirow[b]{2}{*}{ GenBank accession } & \multirow[b]{2}{*}{ GC content (\%) } & \multirow[b]{2}{*}{$\begin{array}{l}\text { Genome density } \\
\text { (genes/kbp) }\end{array}$} & \multicolumn{2}{|c|}{ Phage UFV-P2 } \\
\hline & & & & Identities & $\%$ \\
\hline UFV-P2 & JX863101 & 51.5 & 1.65 & - & - \\
\hline vB_PaeP_p2-10_Or1 & HF543949 & 52.0 & 1.32 & 27,253 & 57.46 \\
\hline vB_PaeP_C1-14_Or & HE983844 & 52.0 & 1.41 & 27,672 & 57.31 \\
\hline LUZ24 & NC_010325 & 52.2 & 1.49 & 27,510 & 56.80 \\
\hline PaP4 & KC294142 & 52.5 & 1.59 & 27,015 & 56.73 \\
\hline $\mathrm{PaP3}$ & NC_004466 & 52.2 & 1.56 & 27,358 & 56.20 \\
\hline MR299-2 & JN254801 & 52.0 & 1.52 & 27,192 & 56.19 \\
\hline Tf & NC_017971 & 53.2 & 1.51 & 23,750 & 49.55 \\
\hline Phi-2 & NC_013638 & 58.9 & 1.00 & 22,672 & 46.73 \\
\hline phiKMV & NC_005045 & 62.3 & 1.13 & 22,392 & 46.43 \\
\hline philBB-PF7A & NC_015264 & 56.3 & 1.27 & 22,150 & 46.41 \\
\hline Bf7 & NC_016764 & 58.4 & 1.15 & 21,825 & 46.16 \\
\hline PaP2 & NC_005884 & 45.4 & 1.32 & 22,523 & 46.11 \\
\hline $119 X$ & NC_007807 & 44.9 & 1.29 & 22,434 & 46.03 \\
\hline T7 (Enterobacteria) & NC_001604 & 48.4 & 1.50 & 21,602 & 45.66 \\
\hline gh-1 & NC_004665 & 57.4 & 1.12 & 21,218 & 45.35 \\
\hline F116 & NC_006552 & 63.2 & 1.07 & 27,102 & 41.20 \\
\hline LUZ7 & NC_013691 & 53.2 & 1.54 & 29,160 & 38.74 \\
\hline
\end{tabular}

coding sequences (CDS) by similarity searches against known proteins in the GenBank and UniProt databases [5]. However, we propose a new annotation of the genome of this virus based on different tools, which were able to functionally predict 75 ORFs also bidirectionally oriented and forming clusters of early and late transcription (Figure 1 and Table 2).

The searches for consensus sequences of transcriptional promoters revealed the presence of seven promoters, five in the positive strand initiating the transcription of ORFs that encode early proteins, which is a common feature of viral genomes that need bacterial transcription factors to start their infection cycle. The two other promoters are located in late genes modules. These genes are usually transcribed by viral transcription factors.

Three rho-independent transcription terminators were predicted using ARNold, one in the positive and two in the negative strand (Figure 1). A bidirectional termination region was found in the region from 25,922 to 25,964 . Interestingly, this pattern of termination is also found in the genomes of the phages PaP3 [1] and LUZ24 [2]. The last terminator sequence is located at the terminal end of the gene encoding the major head protein. The low number of sequences of rho-independent terminators compared to the number of predicted ORFs may be due to the existence of other types of terminators or the presence of transcriptional modules and the generation of polycistronic mRNAs, a very common feature of viral genomes.

The predicted UFV-P2 genes were functionally classified as its promoters, predicted order of transcription, and its annotated functions.

\section{Nucleotide biosynthesis and DNA replication (positive-stranded ORFs)}

Fifty-five genes (ORFs 01-55) involved in nucleotide biosynthesis and viral replication process were found in the UFV-P2 genome positive strand, named early genes (Figure 1). Among viral replication genes, ORF31 encodes a primase/helicase; ORF44, a DNA-binding protein; ORF48, a 5'-3' exonuclease; ORF50, a putative endonuclease; and ORFs 32 and 43 encode the two exons of the viral DNA polymerase, between which there is an ORF encoding a putative holin with three transmembrane domains similar to those from the phages tf and LUZ24. Holins are small membrane proteins that accumulate in the membrane until, at a specific time that is "programmed" into the holin, the membrane suddenly becomes permeabilized to the fully folded endolysin [29]. In addition, the UFV-P2 genome contains two endonucleases encoded by ORF24 and ORF50. The first is a $\mathrm{HNH}$ endonuclease, a group I homing endonuclease, which may be related to the presence of introns in the UFV-P2 genome [30], like those between the two 


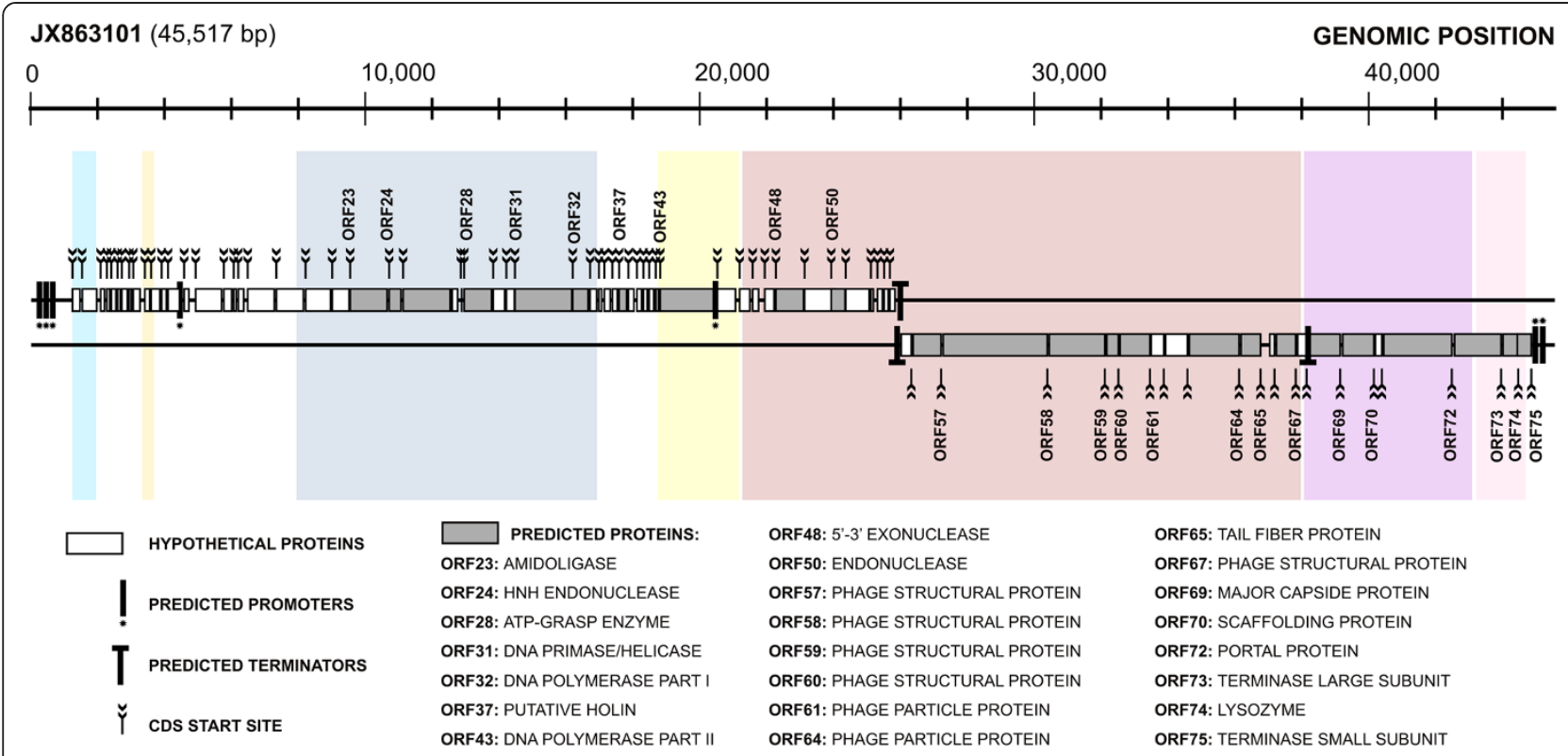

Figure 1 The genomic organization of the phage UFV-P2. The colored squares in the background correspond to the conserved locally collinear blocks (LCB) found for the UFV-P2 in relation to the other members of the Luz24likevirus genus (Figure 2). The detailed annotation is shown in Table 2.

parts of DNA polymerase. Other enzymes predicted in the positive strand include ORFs 23,25 and 28 , which encode, respectively, an amidoligase, a glutamine amidotransferase and an ATP-grasp enzyme. The other 45 proteins of the early genes module are hypothetical proteins.

\section{Virion assembly and host lysis (negative-stranded ORFs)}

Twenty genes (ORFs 56-75) related to the composition and assembly of the viral particle, DNA packaging, and host lysis were found in the UFV-P2 genome negative strand, named late genes (Figure 1). Two transcriptional modules were found based on predicted terminators. The first is located in the regions comprising the ORFs 75-69, and the second module corresponding to the ORFs 75-56.

In the first module, ORF75 and ORF73 encode the small and large terminase subunits, respectively. The terminase is the motor component that assists the translocation of viral genomic DNA to the inner of the capsid during packaging via ATP hydrolysis. There is an ongoing discussion about the role of terminase structure in determining the points for cleavage of the viral DNA, which would influence the entire viral genome organization [31]. Recently, Shen and coworkers [32] functionally identified the two genes encoding PaP3 terminase subunits, located in ORFs 1 and 3, respectively, which have high sequence similarity with ORFs 75 and 73 of the UFV-P2 genome. The PaP3 genome have been annotated as opposing transcriptional gene clusters in relation to the UFV-P2 genome, what explains the difference observed for the numbering of similar ORFs. The same occurred for the earlier annotation of phage UFV-P2 [5], which is revised in this work to correspond to the annotation of phage LUZ24, which represents the genus.

ORF72 encodes the portal protein; ORF69 encodes the major head protein; and ORF70 encodes a scaffolding protein, which is a chaperone possibly related to viral particle assembly. In the second module, beyond the ORFs from the first, the ORFs 57-61, 64 and 67 encode particle/ structural proteins; ORF65 encodes the tail fiber protein; and the other six ORFs encode hypothetical proteins.

ORF74 encodes a lysozyme that is used in the process of host cell breakage through the lysis of the peptidoglycan layer. The occurrence of a lysin, not associated with its cognate holin, is unusual but also found in other members of the Luz24likevirus genus.

\section{Structural genomic comparisons and evolutionary clustering}

Pairwise genomic comparisons has been a useful approach for genotyping and classification of viruses like Circoviridae [33] and Geminiviridae [34]. The alignment of phages genomic sequences and pairwise comparisons revealed that vb_PaeP_p2-10_Or1, vb_PaeP_C114_Or, LUZ24, PaP4, PaP3, MR299-2 and tf are the phages most closely related to UFV-P2. Genomic sequences of these phages presented an identity to the UFV-P2 genome ranging from $49.5 \%$ to $57.5 \%$ (see Table 1 ). 
Table 2 Functional genomic annotation of phage UFV-P2

\begin{tabular}{|c|c|c|c|c|c|c|c|c|}
\hline Gene & Predicted protein & $\begin{array}{l}\text { Genomic } \\
\text { coordinates }\end{array}$ & Strand & $\begin{array}{l}\text { Protein } \\
\text { mass (Da) }\end{array}$ & Protein pl & AA residues & Homologs (a) \& Motifs & E-value \\
\hline ORF01 & $\begin{array}{l}\text { Conserved hypothetical } \\
\text { protein }\end{array}$ & $1205 . .1483$ & + & 10486 & 10.5 & 92 & $\begin{array}{l}\text { NP_955002 hypothetical protein } \\
\text { PaP3p71 [Pseudomonas phage } \\
\text { PaP3] }\end{array}$ & $7.34 \mathrm{E}-09$ \\
\hline ORF02 & $\begin{array}{l}\text { Conserved hypothetical } \\
\text { protein }\end{array}$ & $1501 . .1983$ & + & 17752 & 9.4 & 160 & $\begin{array}{l}\text { YP_006382463 hypothetical protein } \\
\text { tf_02 [Pseudomonas phage tf]; } \\
\text { protein motifs: cl10333 PHA01782 }\end{array}$ & $3.22 \mathrm{E}-37$ \\
\hline ORF03 & $\begin{array}{l}\text { Conserved hypothetical } \\
\text { protein }\end{array}$ & $2046 . .2216$ & + & 6515 & 7.8 & 56 & $\begin{array}{l}\text { YP_007183264 hypothetical protein } \\
\text { BN425_ORF_56 [Pseudomonas } \\
\text { phage vB_PaeP_p2-10_Or1] }\end{array}$ & 7.83E-02 \\
\hline ORF04 & Hypothetical protein & $2231 . .2368$ & + & 5276 & 9.8 & 45 & - & - \\
\hline ORF05 & Hypothetical protein & $2365 . .2556$ & + & 7171 & 9.2 & 63 & - & - \\
\hline ORF06 & $\begin{array}{l}\text { Hypothetical membrane } \\
\text { protein }\end{array}$ & $2553 . .2687$ & + & 4708 & 9.5 & 44 & $\begin{array}{l}\text { protein motifs: one transmembrane } \\
\text { domain discovered using TMHMM } \\
\text { and Phobius }\end{array}$ & \\
\hline ORF07 & $\begin{array}{l}\text { Conserved hypothetical } \\
\text { protein }\end{array}$ & $2677 . .2898$ & + & 8374 & 9.8 & 73 & $\begin{array}{l}\text { YP_006382470 hypothetical protein } \\
\text { tf_10 [Pseudomonas phage tf] }\end{array}$ & $2.09 \mathrm{E}-04$ \\
\hline ORF08 & $\begin{array}{l}\text { Conserved hypothetical } \\
\text { protein }\end{array}$ & 2898.3020 & + & 5178 & 9.3 & 40 & $\begin{array}{l}\text { AGC35239 hypothetical protein } \\
\text { PaP4_008 [Pseudomonas phage } \\
\text { PaP4] }\end{array}$ & $1.19 \mathrm{E}-05$ \\
\hline ORF09 & Hypothetical protein & $3021 . .3275$ & + & 9120 & 4.1 & 84 & - & - \\
\hline ORF10 & Hypothetical protein & $3368 . .3568$ & + & 7493 & 9.6 & 66 & - & - \\
\hline ORF11 & Hypothetical protein & $3555 . .3869$ & + & 11403 & 5.0 & 104 & - & - \\
\hline ORF12 & Hypothetical protein & 3869.4063 & + & 7139 & 9.0 & 64 & - & - \\
\hline ORF13 & $\begin{array}{l}\text { Conserved hypothetical } \\
\text { protein }\end{array}$ & $4060 . .4458$ & + & 14906 & 9.4 & 132 & $\begin{array}{l}\text { YP_006382473 hypothetical protein } \\
\text { tf_13 [Pseudomonas phage tf] }\end{array}$ & $9.55 e-15$ \\
\hline ORF14 & $\begin{array}{l}\text { Hypothetical membrane } \\
\text { protein }\end{array}$ & $4539 . .4736$ & + & 7618 & 9.1 & 65 & $\begin{array}{l}\text { protein motifs: one to two } \\
\text { transmembrane domains discovered } \\
\text { using TMHMM and Phobius }\end{array}$ & \\
\hline ORF15 & $\begin{array}{l}\text { Conserved hypothetical } \\
\text { protein }\end{array}$ & $4884 . .5714$ & + & 29824 & 8.5 & 276 & $\begin{array}{l}\text { AGC35249 transposase fusion } \\
\text { protein [Pseudomonas phage PaP4]; } \\
\text { protein motifs: pfam01145 Band_7, } \\
\text { \& cl02525 Band_7 }\end{array}$ & 1.15E-113 \\
\hline ORF16 & $\begin{array}{l}\text { Conserved hypothetical } \\
\text { protein }\end{array}$ & $5727 . .6017$ & + & 10708 & 9.1 & 96 & $\begin{array}{l}\text { YP_006659979 hypothetical protein } \\
\text { tf_14 [Pseudomonas phage tf] }\end{array}$ & $2.29 \mathrm{E}-11$ \\
\hline ORF17 & $\begin{array}{l}\text { Conserved hypothetical } \\
\text { protein }\end{array}$ & $6021 . .6149$ & + & 4783 & 11.0 & 42 & $\begin{array}{l}\text { NP_775206 hypothetical protein } \\
\text { PaP3p51 [Pseudomonas phage PaP3] }\end{array}$ & $1.32 \mathrm{E}-03$ \\
\hline ORF18 & Hypothetical protein & $6159 . .6374$ & + & 7803 & 5.0 & 71 & - & - \\
\hline ORF19 & $\begin{array}{l}\text { Conserved hypothetical } \\
\text { protein }\end{array}$ & $6441 . .7289$ & + & 31291 & 9.2 & 282 & $\begin{array}{l}\text { YP_006382477 hypothetical protein } \\
\text { tf_17 [Pseudomonas phage tf] }\end{array}$ & $1.75 \mathrm{E}-03$ \\
\hline ORF20 & $\begin{array}{l}\text { Conserved hypothetical } \\
\text { protein }\end{array}$ & $7296 . .8159$ & + & 32906 & 4.9 & 287 & $\begin{array}{l}\text { YP_006382478 hypothetical protein } \\
\text { tf_18 [Pseudomonas phage tf] }\end{array}$ & 2.06E-07 \\
\hline ORF21 & $\begin{array}{l}\text { Conserved hypothetical } \\
\text { protein }\end{array}$ & $8171 . .8968$ & + & 29423 & 5.0 & 265 & $\begin{array}{l}\text { YP_006382479 hypothetical protein } \\
\text { tf_19 [Pseudomonas phage tf]; } \\
\text { protein motifs: PF14395.1 } \\
\text { COOH-NH2_lig }\end{array}$ & $8.75 \mathrm{E}-112$ \\
\hline ORF22 & $\begin{array}{l}\text { Conserved hypothetical } \\
\text { protein }\end{array}$ & $8965 . .9525$ & + & 21203 & 7.5 & 186 & $\begin{array}{l}\text { NP__775210 hypothetical protein } \\
\text { PaP3p47 [Pseudomonas phage } \\
\text { PaP3] }\end{array}$ & $2.53 \mathrm{E}-40$ \\
\hline ORF23 & Amidoligase & $9501 . .10658$ & + & 43407 & 5.5 & 385 & $\begin{array}{l}\text { YP_001671891 hypothetical protein } \\
\text { [Pseudomonas phage LUZ24]; protein } \\
\text { motifs: PF12224.3 Amidoligase_2 }\end{array}$ & 4.46E-96 \\
\hline ORF24 & HNH endonuclease & 10667..11071 & + & 15340 & 9.6 & 134 & $\begin{array}{l}\text { YP_002003475 gp2.8 [Enterobacteria } \\
\text { phage BA14]; protein motifs: PF13392.1 } \\
\text { HNH_3 }\end{array}$ & 2.71E-20 \\
\hline
\end{tabular}


Table 2 Functional genomic annotation of phage UFV-P2 (Continued)

\begin{tabular}{|c|c|c|c|c|c|c|c|c|}
\hline ORF25 & $\begin{array}{l}\text { Glutamine } \\
\text { amidotransferase }\end{array}$ & $11084 . .12544$ & + & 54036 & 5.9 & 486 & $\begin{array}{l}\text { YP_006382482 glutamine } \\
\text { amidotransferase [Pseudomonas } \\
\text { phage tf]; protein motifs: PF13522.1 } \\
\text { GATase_6, \& cd00352 Gn_AT_II }\end{array}$ & $2.72 \mathrm{E}-151$ \\
\hline ORF26 & $\begin{array}{l}\text { Conserved hypothetical } \\
\text { protein }\end{array}$ & $12547 . .12765$ & + & 8166 & 4.8 & 72 & $\begin{array}{l}\text { NP_775213 hypothetical protein } \\
\text { PaP3p44 [Pseudomonas phage PaP3] }\end{array}$ & $2.25 \mathrm{E}-20$ \\
\hline ORF27 & Hypothetical protein & $12805 . .12909$ & + & 3505 & 5.3 & 34 & - & - \\
\hline ORF28 & ATP-grasp enzyme & $12906 . .13781$ & + & 31797 & 7.7 & 291 & $\begin{array}{l}\text { YP_006382486 hypothetical protein } \\
\text { tf_24 [Pseudomonas phage tf] }\end{array}$ & $2.60 \mathrm{E}-96$ \\
\hline ORF29 & $\begin{array}{l}\text { Conserved hypothetical } \\
\text { protein }\end{array}$ & 13774..14169 & + & 15076 & 5.5 & 131 & $\begin{array}{l}\text { AGC35259 hypothetical protein } \\
\text { PaP4_028 [Pseudomonas phage } \\
\text { PaP4]; proteins motifs: PF06094.7 } \\
\text { AIG2, \& cd06661 GGCT_like }\end{array}$ & $5.55 E-27$ \\
\hline ORF30 & $\begin{array}{l}\text { Conserved hypothetical } \\
\text { protein }\end{array}$ & $14169 . .14465$ & + & 11295 & 5.4 & 98 & $\begin{array}{l}\text { YP_006382488 hypothetical protein } \\
\text { tf_026 [Pseudomonas phage tf] }\end{array}$ & $6.03 \mathrm{E}-23$ \\
\hline ORF31 & DNA primase/helicase & $14425 . .16176$ & + & 65673 & 5.9 & 583 & $\begin{array}{l}\text { YP_001671897 primase/helicase } \\
\text { [Pseudomonas phage LUZ24]; protein } \\
\text { motifs: PF13155.1 Toprim_2, \& } \\
\text { PF03796.10 DnaB_C }\end{array}$ & 0 \\
\hline ORF32 & DNA polymerase part I & $16151 . .16666$ & + & 20133 & 4.5 & 171 & $\begin{array}{l}\text { YP_006382490 3'-5' exonuclease } \\
\text { [Pseudomonas phage tf] \& } \\
\text { YP_001671898 DNA polymerase } \\
\text { part I [Pseudomonas phage LUZ24] }\end{array}$ & $\begin{array}{l}2.82 \mathrm{E}-75 \\
3.72 \mathrm{e}-68\end{array}$ \\
\hline ORF33 & $\begin{array}{l}\text { Conserved hypothetical } \\
\text { membrane protein }\end{array}$ & $16669 . .16920$ & + & 9085 & 10.0 & 83 & $\begin{array}{l}\text { YP_006382491 hypothetical protein } \\
\text { tf_29 [Pseudomonas phage tf]; protein } \\
\text { motifs: one transmembrane domain } \\
\text { discovered using TMHMM and Phobius }\end{array}$ & $1.99 \mathrm{E}-06$ \\
\hline ORF34 & Hypothetical protein & $16931 . .17068$ & + & 5096 & 3.4 & 45 & - & - \\
\hline ORF35 & Hypothetical protein & $17095 . .17325$ & + & 8505 & 9.0 & 76 & - & - \\
\hline ORF36 & $\begin{array}{l}\text { Conserved hypothetical } \\
\text { protein }\end{array}$ & $17336 . .17539$ & + & 7596 & 5.8 & 67 & $\begin{array}{l}\text { YP_001671900 hypothetical protein } \\
\text { [Pseudomonas phage LUZ24] }\end{array}$ & $2.23 \mathrm{E}-02$ \\
\hline ORF37 & Putative holin & $17536 . .17817$ & + & 10475 & 9.8 & 93 & $\begin{array}{l}\text { YP_006382493 hypothetical protein } \\
\text { tf_32 [Pseudomonas phage tf] \& } \\
\text { YP_001671904 putative holin } \\
\text { [Pseudomonas phage LUZ24]; } \\
\text { protein motifs: three transmembrane } \\
\text { domains discovered using TMHMM } \\
\text { and Phobius }\end{array}$ & $\begin{array}{l}\text { 5.03e-35; } \\
1.92 \mathrm{e}-18\end{array}$ \\
\hline ORF38 & $\begin{array}{l}\text { Hypothetical membrane } \\
\text { protein }\end{array}$ & $17814 . .18032$ & + & 8079 & 9.9 & 72 & $\begin{array}{l}\text { protein motifs: one or two } \\
\text { transmembrane domains discovered } \\
\text { using TMHMM and Phobius }\end{array}$ & \\
\hline ORF39 & $\begin{array}{l}\text { Conserved hypothetical } \\
\text { protein }\end{array}$ & $18069 . .18263$ & + & 6728 & 3.5 & 64 & $\begin{array}{l}\text { YP_006382494 hypothetical protein } \\
\text { tf_34 [Pseudomonas phage tf] }\end{array}$ & $6.48 \mathrm{E}-04$ \\
\hline ORF40 & $\begin{array}{l}\text { Conserved hypothetical } \\
\text { protein }\end{array}$ & $18263 . .18433$ & + & 5628 & 8.2 & 56 & $\begin{array}{l}\text { YP_007112538 hypothetical protein } \\
\text { MAR_61 [Vibrio phage vB_VpaM_MAR] }\end{array}$ & $9.20 \mathrm{E}-05$ \\
\hline ORF41 & $\begin{array}{l}\text { Conserved hypothetical } \\
\text { protein }\end{array}$ & 18433...18627 & + & 7272 & 10.9 & 64 & $\begin{array}{l}\text { YP_006382495 hypothetical protein } \\
\text { tf_35 [Pseudomonas phage tf] }\end{array}$ & $1.52 \mathrm{E}-03$ \\
\hline ORF42 & Hypothetical protein & 18630..18764 & + & 4887 & 6.0 & 44 & - & - \\
\hline ORF43 & DNA polymerase part II & $18765 . .20405$ & + & 60865 & 9.6 & 546 & $\begin{array}{l}\text { YP_007183240 DNA polymerase1 } \\
\text { [Pseudomonas phage vB_PaeP } \\
\text { p2-10_Or1] \& YP_001671907 DNA } \\
\text { polymerase part II [Pseudomonas } \\
\text { phage LUZ24]; protein motifs: } \\
\text { PF00476.15 DNA_pol_A }\end{array}$ & $0 ; 0$ \\
\hline ORF44 & DNA binding protein & $20472 . .21068$ & + & 21853 & 4.8 & 198 & $\begin{array}{l}\text { YP_006382500 DNA binding protein } \\
\text { [Pseudomonas phage tf] }\end{array}$ & $6.61 \mathrm{E}-70$ \\
\hline ORF45 & $\begin{array}{l}\text { Conserved hypothetical } \\
\text { protein }\end{array}$ & $21139 . .21495$ & + & 13200 & 6.3 & 118 & $\begin{array}{l}\text { AGC35272 hypothetical protein } \\
\text { PaP4_041 [Pseudomonas phage PaP4] }\end{array}$ & $4.02 \mathrm{E}-47$ \\
\hline
\end{tabular}


Table 2 Functional genomic annotation of phage UFV-P2 (Continued)

\begin{tabular}{|c|c|c|c|c|c|c|c|c|}
\hline ORF46 & Hypothetical protein & $21525 . .21755$ & + & 8335 & 4.7 & 76 & - & - \\
\hline ORF47 & Hypothetical protein & $21887 . .22222$ & + & 12649 & 9.4 & 111 & - & - \\
\hline ORF48 & $5^{\prime}-3^{\prime}$ exonuclease & $22219 . .23103$ & + & 33498 & 5.3 & 294 & $\begin{array}{l}\text { NP_775229 exonuclease [Pseudomonas } \\
\text { phage PaP3]; protein motifs: PF01367.15 } \\
\text { 5_3_exonuc, \& cd09898 H3TH_53EXO }\end{array}$ & $1.47 \mathrm{E}-149$ \\
\hline ORF49 & $\begin{array}{l}\text { Conserved hypothetical } \\
\text { protein }\end{array}$ & $23078 . .24073$ & + & 37154 & 4.7 & 331 & $\begin{array}{l}\text { YP_006659984 conserved hypothetical } \\
\text { protein [Pseudomonas phage tf] }\end{array}$ & $1.15 E-39$ \\
\hline ORF50 & Endonuclease & $23874 . .24341$ & + & 17655 & 5.4 & 155 & $\begin{array}{l}\text { YP_006382505 endonuclease } \\
\text { [Pseudomonas phage tf] }\end{array}$ & $1.11 \mathrm{E}-41$ \\
\hline ORF51 & $\begin{array}{l}\text { Conserved hypothetical } \\
\text { protein }\end{array}$ & $24307 . .25062$ & + & 28981 & 6.2 & 251 & $\begin{array}{l}\text { YP_001671917 hypothetical protein } \\
\text { [Pseudomonas phage LUZ24] }\end{array}$ & 2.15E-129 \\
\hline ORF52 & Hypothetical protein & $25059 . .25184$ & + & 4844 & 10.5 & 41 & - & - \\
\hline ORF53 & $\begin{array}{l}\text { Conserved hypothetical } \\
\text { protein }\end{array}$ & $25255 . .25458$ & + & 7717 & 6.5 & 67 & $\begin{array}{l}\text { YP_006659986 hypothetical protein } \\
\text { tf_48 [Pseudomonas phage tf] }\end{array}$ & 7.14E-14 \\
\hline ORF54 & Hypothetical protein & $25455 . .25631$ & + & 6814 & 4.6 & 58 & - & - \\
\hline ORF55 & $\begin{array}{l}\text { Conserved hypothetical } \\
\text { protein }\end{array}$ & $25628 . .25834$ & + & 8109 & 4.4 & 68 & $\begin{array}{l}\text { YP_001671920 hypothetical protein } \\
\text { [Pseudomonas phage LUZ24] }\end{array}$ & $1.57 \mathrm{E}-12$ \\
\hline ORF56 & $\begin{array}{l}\text { Conserved hypothetical } \\
\text { protein }\end{array}$ & $25963 . .26319$ & - & 13124 & 5.9 & 118 & $\begin{array}{l}\text { AGC35282 hypothetical protein } \\
\text { PaP4_051 [Pseudomonas phage PaP4] }\end{array}$ & $2.11 \mathrm{E}-45$ \\
\hline ORF57 & Phage structural protein & $26321 . .27208$ & - & 32029 & 5.5 & 295 & $\begin{array}{l}\text { AFD10698 hypothetical protein } \\
\text { I7C_020 [Pseudomonas phage } \\
\text { MR299-2] }\end{array}$ & 3.43E-152 \\
\hline ORF58 & Phage structural protein & $27219 . .30383$ & - & 111420 & 5.3 & 1054 & $\begin{array}{l}\text { YP_001671923 phage particle protein } \\
\text { [Pseudomonas phage LUZ24] }\end{array}$ & 0 \\
\hline ORF59 & Phage structural protein & $30389 . .32101$ & - & 60235 & 5.5 & 570 & $\begin{array}{l}\text { YP_001671924 phage particle protein } \\
\text { [Pseudomonas phage LUZ24] }\end{array}$ & $1.35 \mathrm{E}-86$ \\
\hline ORF60 & Phage structural protein & $32103 . .32507$ & - & 13485 & 6.5 & 134 & $\begin{array}{l}\text { YP_001671925 phage particle protein } \\
\text { [Pseudomonas phage LUZ24] }\end{array}$ & $9.26 \mathrm{E}-11$ \\
\hline ORF61 & Phage particle protein & $32504 . .33448$ & - & 32240 & 4.8 & 314 & $\begin{array}{l}\text { AFD10694 hypothetical protein } \\
\text { I7C_016 [Pseudomonas phage } \\
\text { MR299-2] }\end{array}$ & 3.23E-112 \\
\hline ORF62 & $\begin{array}{l}\text { Conserved hypothetical } \\
\text { protein }\end{array}$ & $33429 . .33863$ & - & 16522 & 4.5 & 144 & $\begin{array}{l}\text { AGC35288 hypothetical protein } \\
\text { PaP4_057 [Pseudomonas phage PaP4] }\end{array}$ & $2.95 \mathrm{E}-57$ \\
\hline ORF63 & $\begin{array}{l}\text { Conserved hypothetical } \\
\text { protein }\end{array}$ & $33860 . .34570$ & - & 25585 & 4.8 & 236 & $\begin{array}{l}\text { YP_001671928 hypothetical protein } \\
\text { [Pseudomonas phage LUZ24] }\end{array}$ & $3.73 \mathrm{E}-56$ \\
\hline ORF64 & Phage particle protein & $34570 . .36111$ & - & 57542 & 5.1 & 513 & $\begin{array}{l}\text { YP_006382515 phage particle protein } \\
\text { [Pseudomonas phage tf] \& } \\
\text { YP_001671929 hypothetical protein } \\
\text { [Pseudomonas phage LUZ24] }\end{array}$ & $0 ; 0$ \\
\hline ORF65 & Tail fiber protein & $36119 . .36754$ & - & 21879 & 6.7 & 211 & $\begin{array}{l}\text { NP_775246 hypothetical protein } \\
\text { PaP3p12 [Pseudomonas phage PaP3] }\end{array}$ & $1.68 \mathrm{E}-62$ \\
\hline ORF66 & $\begin{array}{l}\text { Conserved hypothetical } \\
\text { protein }\end{array}$ & $36976 . .37170$ & - & 6838 & 6.6 & 64 & $\begin{array}{l}\text { YP_001671932 hypothetical protein } \\
\text { LUZ24 [Pseudomonas phage LUZ24] }\end{array}$ & $3.02 \mathrm{E}-28$ \\
\hline ORF67 & phage structural protein & $37175 . .37804$ & - & 23887 & 5.0 & 209 & $\begin{array}{l}\text { AFD10687 putative constituent protein } \\
\text { [Pseudomonas phage MR299-2] }\end{array}$ & $2.54 \mathrm{E}-82$ \\
\hline ORF68 & $\begin{array}{l}\text { Conserved hypothetical } \\
\text { protein }\end{array}$ & $37808 . .38128$ & - & 11847 & 5.8 & 106 & $\begin{array}{l}\text { YP_007183215 hypothetical protein } \\
\text { BN425_ORF_07 [Pseudomonas phage } \\
\text { VB_PaeP_p2-1 0_Or1] }\end{array}$ & $1.26 \mathrm{E}-50$ \\
\hline ORF69 & Major capsid protein & $38180 . .39133$ & - & 34740 & 6.0 & 317 & $\begin{array}{l}\text { NP_775251 major head protein } \\
\text { [Pseudomonas phage PaP3] \& } \\
\text { YP_001671935 major head protein } \\
\text { [Pseudomonas phage LUZ24] }\end{array}$ & $0 ; 0$ \\
\hline ORF70 & Scaffolding protein & $39152 . .40138$ & - & 36190 & 4.3 & 328 & $\begin{array}{l}\text { YP_001671936 scaffolding protein } \\
\text { [Pseudomonas phage LUZ24] }\end{array}$ & 4.90E-101 \\
\hline
\end{tabular}


Table 2 Functional genomic annotation of phage UFV-P2 (Continued)

\begin{tabular}{|c|c|c|c|c|c|c|c|c|}
\hline ORF71 & $\begin{array}{l}\text { Conserved hypothetical } \\
\text { protein }\end{array}$ & 40128.40379 & - & 9559 & 5.2 & 83 & $\begin{array}{l}\text { AGC35298 hypothetical protein } \\
\text { PaP4_067 [Pseudomonas phage } \\
\text { PaP4] \& YP_001671937 hypothetical } \\
\text { protein [Pseudomonas phage LUZ24] }\end{array}$ & $\begin{array}{l}\text { 8.9E-30; } \\
2.00 \mathrm{e}-29\end{array}$ \\
\hline ORF72 & Portal protein & $40379 . .42469$ & - & 79405 & 5.0 & 696 & $\begin{array}{l}\text { YP_007183212 putative portal protein } \\
\text { [Pseudomonas phage vB_PaeP_- } \\
\text { p2-10_Or1]YP_001671938 portal } \\
\text { protein [Pseudomonas phage LUZ24] }\end{array}$ & $0 ; 0$ \\
\hline ORF73 & Terminase, large subunit & 42496.43941 & - & 54330 & 6.0 & 481 & $\begin{array}{l}\text { YP_001671939 terminase large subunit } \\
\text { [Pseudomonas phage LUZ24]; protein } \\
\text { motifs: PF03237.10 Terminase_6 }\end{array}$ & 0 \\
\hline ORF74 & Lysozyme & 43945.44451 & - & 18961 & 8.6 & 168 & $\begin{array}{l}\text { YP_006382529 lysozyme [Pseudomonas } \\
\text { phage tf]; protein motifs: PF00959.14 } \\
\text { Phage_lysozyme }\end{array}$ & $3.27 E-56$ \\
\hline ORF75 & Terminase, small subunit & $44372 . .44845$ & - & 17291 & 5.9 & 157 & $\begin{array}{l}\text { YP_007183209 hypothetical protein } \\
\text { BN425_ORF_01 [Pseudomonas phage } \\
\text { vB_PaeP_p2-1 0_Or1] \& YP_006382530 } \\
\text { terminase small subunit [Pseudomonas } \\
\text { phage tf] }\end{array}$ & $\begin{array}{l}\text { 5.76E-66; } \\
2.59 \mathrm{e}-64\end{array}$ \\
\hline
\end{tabular}

The structural genomic comparisons in Mauve showed that these phages shared a set of conserved locally collinear blocks (LCB) (Figure 2 and Additional file 1: Figure S2). LCBs are conserved segments that appear be free from genome rearrangements, since the orthologous regions of genomes can be reordered or inverted by recombination processes [21]. In addition, a specific comparison between UFV-P2 and LUZ24 showed colinearity across their genomes (Figures 2 and 3).

Phages LUZ24, PaP4, and UFV-P2 present a conserved bidirectional genomic organization, which is showed by the shared LCBs (blocks 3-9) (Figure 2). Phage tf also presents this organization, but with some differences in the shared LCBs. On the other hand, phages MR299-2, PaP3, vb_PaeP_p2-10_Or1, and vb_PaeP_C114_Or present an inverted set of LCBs (blocks 9-3), representing an opposing arrangement of the gene modules. Proteins of these seven phages were the top hits with the UFV-P2 sequences (Table 2) and can collaborate with each other's functional annotations. In addition to genomic comparisons, a search for direct terminal repeats (DTRs) indicated the presence of patterns at the ends of

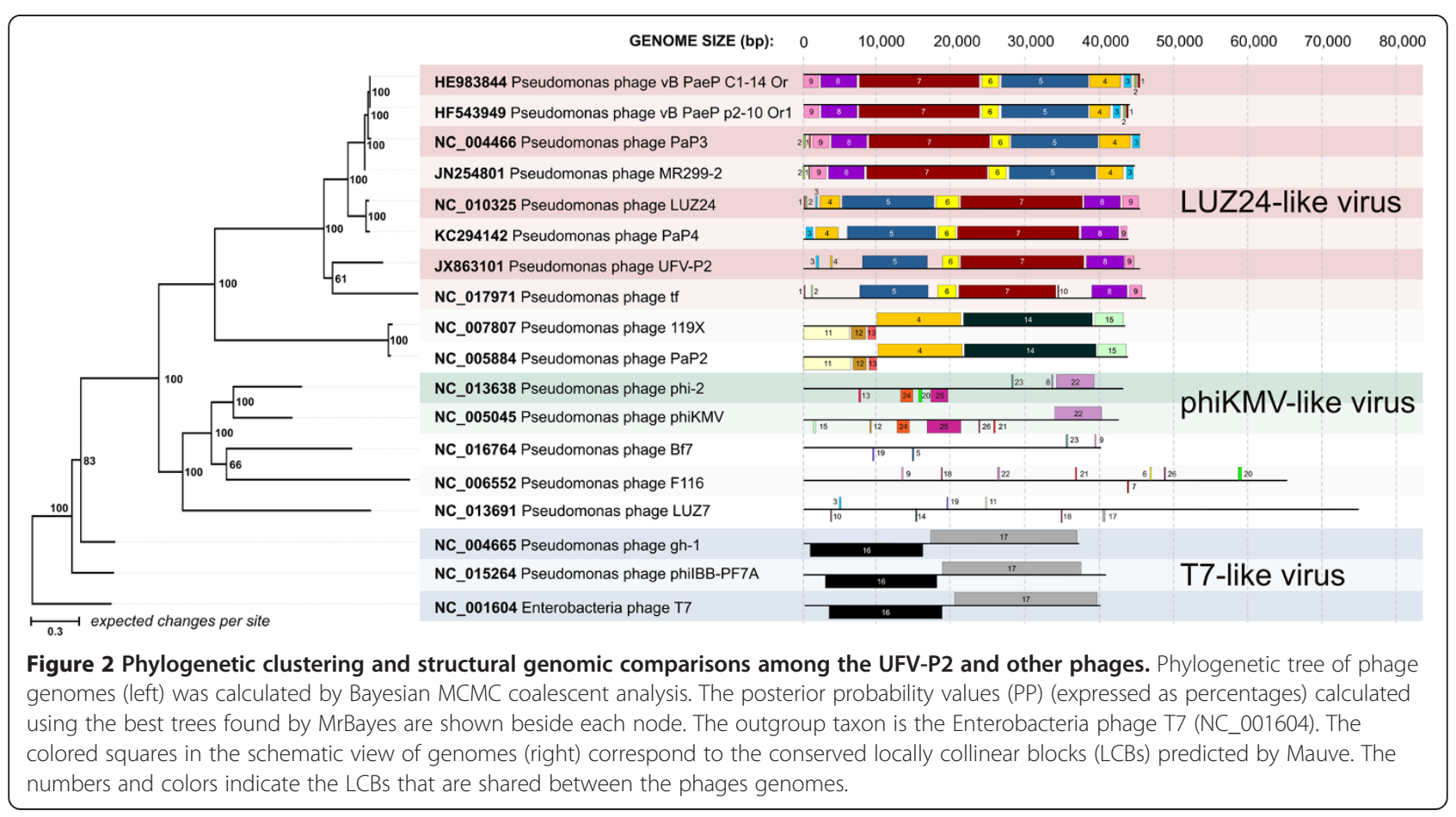




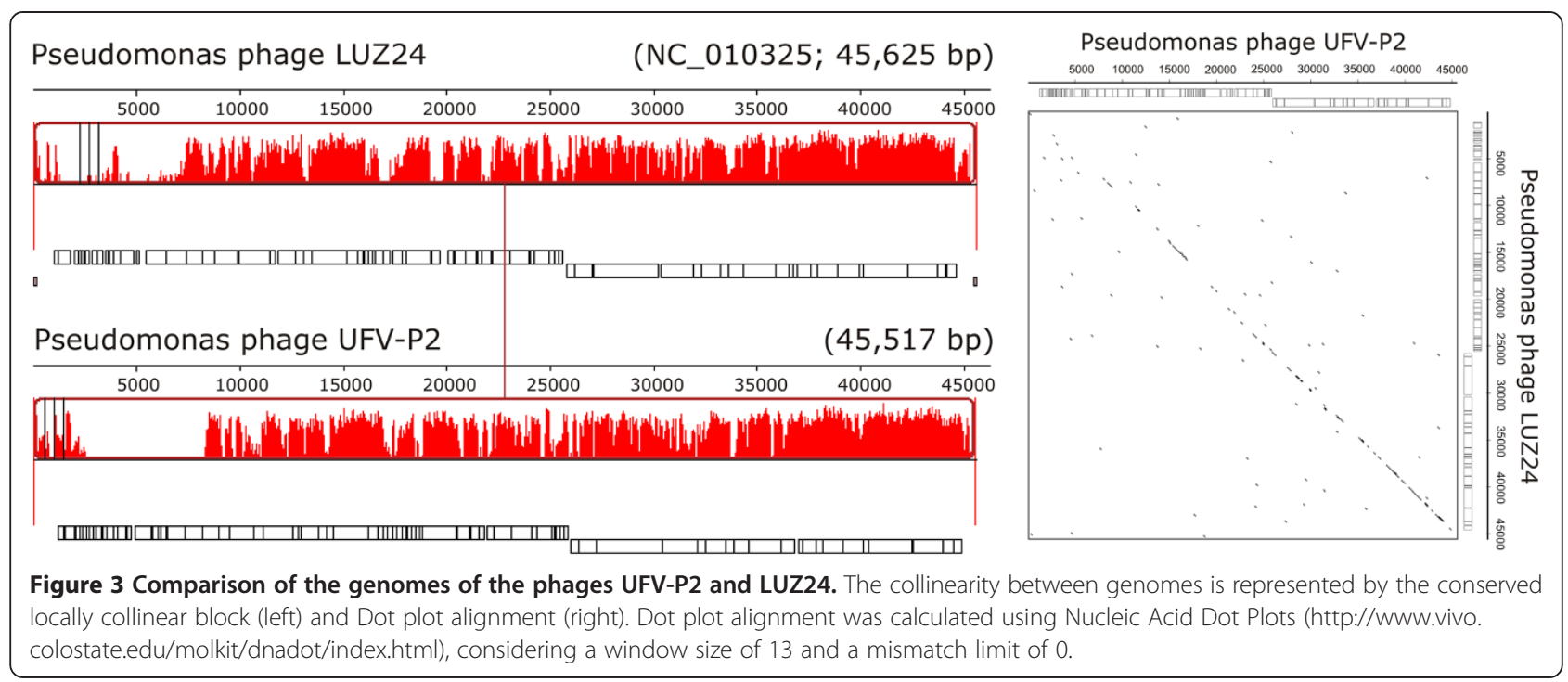

the UFV-P2 genome, as described for the phages LUZ24, tf, and vB_PaeP_C1-14_Or1. These repeats are responsible for the recognition and cleavage of the phage genome at the end of the repeat region during packaging. Interestingly, one of the unique features of this group of phages is that PaP3 possesses 20 bp $5^{\prime}$-protuding cohesive ends [1], while LUZ24 has 184 bp DTRs, yet there does not appear to be a significant difference in the amino acid sequence of their terminases.

As suggested by the structural genomic comparisons, phylogenetic tree of genomic sequences grouped the phages according the shared LCBs (Figure 2). Phages PaeP_p2-10_Or1, vb_PaeP_C1-14_Or, LUZ24, PaP4, PaP3, MR299-2, tf, and UFV-P2 were included in a distinct monophyletic clade in BI phylogenetic tree, which possibly represents the Luz24likevirus genus. The shared LCBs, blocks 3-9 (Figure 2), may be considered as a genomic signature for this genus. In UFV-P2 genome (Figure 1), as for the other phages, the genes for biosynthesis and DNA replication are included in blocks 5 and 6; genes for virion structure and assembly are in blocks 7 and 8; and genes for host lysis are block 9. In blocks 3 and 4 are included only hypothetical genes. Then, we propose the classification of the phage UFV-P2 in the Luz24likevirus genus. In fact, these analyzes showed that other viruses were also grouped in distinct monophyletic clades or according to specific shared locally collinear blocks (LCB), as those from the T7likevirus (blocks 16 and 17) and Phikmvlikevirus (blocks 22, 24, and 25) genera, beyond a possibly genus including the phages $\mathrm{PaP} 2$ and 199X (blocks 4 and 11-15).

\section{Conclusions}

We have presented the functional annotation of UFV-P2, a new Pseudomonas fluorescens phage. Based on structural genomic comparison and phylogenetic clustering, we suggest the classification of UFV-P2 in the Luz24likevirus genus, and present a set of shared locally collinear blocks as the genomic signature for this genus.

\section{Additional file}

Additional file 1: Figure S1. Transmission Electron Microscopy of the phage UFV-P2. Virions have isometric capsids of $40-50 \mathrm{~nm}$ and very short tails (arrows). Scale bars $=100 \mathrm{~nm}$. Figure S2. Comparison of the genomes of phages classified in LUZ24likevirus genus. The collinearity among genomes is represented by the conserved locally collinear blocks (LCBs). In the main block (blue), the regions of similarity plot with high identity corresponds to the set of shared LCBs (see Figure 2). The connection line between blocks correspond to the central point of LCB of the reference genome (phage LUZ24 genome).

\section{Competing interests}

The authors declare that they have no competing interests.

\section{Authors' contributions}

MRE carried out the phage isolation, propagation, DNA extration, participated in the sequence alignment and drafted the manuscript. PMPV and RLS carried out the bioinformatic analysis and drafted the manuscript. MPA and RSD participated in the design of the study and initial processes of phage isolation, propagation, DNA extration and analysis. CCS and AFC were essential on the concept of the study, and participated in its design and coordination. AK participated in the bioinformatic analysis and concepts of phage classification. SOP is the responsible for the design and coordination of the project. All authors read and approved the final manuscript.

\section{Acknowledgments}

This study was supported by grants from the Fundação de Amparo à Pesquisa do Estado de Minas Gerais (FAPEMIG), Coordenação de Aperfeiçoamento de Pessoal de Nível Superior (CAPES) and Conselho Nacional de Desenvolvimento Científico e Tecnológico (CNPq). The funders had no role in the study design, data collection, analysis, decision to publish, or preparation of this manuscript.

\section{Author details}

'Department of Food Technology, Federal University of Viçosa, Av. PH Rolfs, s/n, Campus da UFV, Viçosa, Minas Gerais 36570-000, Brazil. "Bioinformatics Laboratory, Institute of Applied Biotechnology to Agriculture (BIOAGRO), 
Federal University of Viçosa, Av. PH Rolfs, s/n, Campus da UFV, Viçosa, Minas Gerais 36570-000, Brazil. ${ }^{3}$ Department of Biochemistry and Molecular Biology, Federal University of Viçosa, Av. PH Rolfs, s/n, Campus da UFV, Viçosa, Minas Gerais 36570-000, Brazil. ${ }^{4}$ Laboratory of Molecular Immunovirology, Federal University of Viçosa, Av. PH Rolfs, s/n, Campus da UFV, Viçosa, Minas Gerais 36570-000, Brazil. ${ }^{5}$ Department of General Biology, Federal University of Viçosa, Av. PH Rolfs, s/n, Campus da UFV, Viçosa, Minas Gerais 36570-000, Brazil. ${ }^{6}$ Department of Microbiology, Federal University of Viçosa, Av. PH Rolfs, s/n, Campus da UFV, Viçosa, Minas Gerais 36570-000, Brazil. ${ }^{7}$ Public Health Agency of Canada, Laboratory for Foodborne Zoonoses, Guelph, Ontario N1G 3 W4, Canada. ${ }^{8}$ Department of Molecular and Cellular Biology, University of Guelph, Guelph, Ontario N1G 2 W1, Canada.

Received: 16 July 2013 Accepted: 26 December 2013

Published: 3 January 2014

\section{References}

1. Tan Y, Zhang K, Rao X, Jin X, Huang J, Zhu J, Chen Z, Hu X, Shen X, Wang L, Hu F: Whole genome sequencing of a novel temperate bacteriophage of $P$. aeruginosa: evidence of tRNA gene mediating integration of the phage genome into the host bacterial chromosome. Cell Microbiol 2007, 9:479-491.

2. Ceyssens P-J, Hertveldt K, Ackermann H-W, Noben J-P, Demeke M, Volckaert G, Lavigne R: The intron-containing genome of the lytic Pseudomonas phage LUZ24 resembles the temperate phage PaP3. Virology 2008, 377:233-238.

3. Kulakov LA, Kochetkov W, Ksenzenko VN, Krylov VN, Boronin AM: [Physical map of the DNA of bacteriophage $t f$ of Pseudomonas putida]. Mol Gen Mikrobiol Virusol 1988(6):12-16.

4. Alemayehu D, Casey PG, McAuliffe O, Guinane CM, Martin JG, Shanahan F, Coffey A, Ross RP, Hill C: Bacteriophages $\varphi \mathrm{MR} 299-2$ and $\varphi \mathrm{NH}-4$ can eliminate Pseudomonas aeruginosa in the murine lung and on cystic fibrosis lung airway cells. mBio 2012, 3:e00029-12.

5. Eller MR, Salgado RL, Vidigal PMP, Alves MP, Dias RS, De Oliveira LL, Da Silva CC, De Carvalho AF, De Paula SO: Complete genome sequence of the Pseudomonas fluorescens bacteriophage UFV-P2. Genome Announcement 2013, 1:1 e00006-12.

6. Rasolofo EA, St-Gelais D, LaPointe G, Roy D: Molecular analysis of bacterial population structure and dynamics during cold storage of untreated and treated milk. Int J Food Microbiol 2010, 138:108-118.

7. Munsch-Alatossava P, Alatossava T: Phenotypic characterization of raw milk-associated psychrotrophicbacteria. Microbiol Res 2006, 161:334-346.

8. Baruzzi F, Lagonigro R, Quintieri L, Morea M, Caputo L: Occurrence of non-lactic acid bacteria populations involved in protein hydrolysis of cold-stored high moisture Mozzarella cheese. Food Microbiol 2012, 30:37-44.

9. Arcuri EF, Aparecida M, Paiva V, Lange CC: Contagem, isolamento e caracterização de bactériaspsicrotróficascontaminantes de leite cru refrigerado. Ciência Rural 2008, 38:2250-2255.

10. Dogan B, Boor KJ: Genetic diversity and spoilage potentials among Pseudomonas spp. isolated from fluid milk products and dairy processing plants. Appl Environ Microbiol 2003, 69:130-138.

11. Baum MM, Kainović A, O'Keeffe T, Pandita R, McDonald K, Wu S, Webster P: Characterization of structures in biofilms formed by a Pseudomonas fluorescensisolated from soil. BMC Microbiol 2009, 9:103.

12. Sillankorva S, Neubauer $P$, Azeredo J: Isolation and characterization of a T7-like lytic phage for Pseudomonas fluorescens. BMC Biotechnol 2008, 8:80.

13. Sillankorva $S$, Neubauer P, Azeredo J: Pseudomonas fluorescens biofilms subjected to phage philBB-PF7A. BMC Biotechnol 2008, 08:79.

14. Lingohr EJ, Villegas A, She Y-M, Ceyssens P-J, Kropinski AM: The genome and proteome of the Kluyverabacteriophage Kvp1-another member of the T7-like Autographivirinae. Virol J 2008, 5:122.

15. Sonnhammer EL, Von Heijne G, Krogh A: A hidden Markov model for predicting transmembrane helices in protein sequences.Proceedings/. International Conference on Intelligent Systems for Molecular Biology; ISMB International Conference on Intelligent Systems for. Mol Biol 1998 6:175-182.

16. Käll L, Krogh A, Sonnhammer ELL: A combined transmembrane topology and signal peptide prediction method. J Mol Biol 2004, 338:1027-1036.
17. Derbyshire MK, Lanczycki CJ, Bryant SH, Marchler-Bauer A: Annotation of functional sites with the Conserved Domain Database. Database (Oxford) 2012, 2012:bar058.

18. Macke TJ, Ecker DJ, Gutell RR, Gautheret D, Case DA, Sampath R: RNAMotif, an RNA secondary structure definition and search algorithm. Nucleic Acids Res 2001, 29:4724-4735.

19. Gautheret D, Lambert A: Direct RNA motif definition and identification from multiple sequence alignments using secondary structure profiles. J Mol Biol 2001, 313:1003-1011.

20. Rice P, Longden I, Bleasby A: EMBOSS: the European Molecular Biology Open Software Suite. TIG 2000, 16:276-277.

21. Darling $A E$, Mau B, Perna NT: progressiveMauve: multiple genome alignment with gene gain, loss and rearrangement. PloS one 2010, 5:e11147.

22. Mahadevan P, King JF, Seto D: Data mining pathogen genomes using GeneOrder and CoreGenes and CGUG: gene order, synteny and in silicoproteomes. Int J Comput Biol Drug Des 2009, 2:100-114.

23. Kropinski AM, Borodovsky M, Carver TJ, Cerdeño-Tárraga AM, Darling A, Lomsadze A, Mahadevan P, Stothard P, Seto D, Van Domselaar G, Wishart DS: In silico identification of genes in bacteriophage DNA. Methods Mol Biol 2009, 502:57-89.

24. Ronquist F, Teslenko M, van der Mark P, Ayres DL, Darling A, Hohna S, Larget B, Liu L, Suchard MA, Huelsenbeck JP: MrBayes 3.2: efficient Bayesian phylogenetic inference and model choice across a large model space. Syst Biol 2012, 61(3):539-542

25. Thompson JD, Higgins DG, Gibson TJ: CLUSTAL W: improving the sensitivity of progressive multiple sequence alignment through sequence weighting, position-specific gap penalties and weight matrix choice. Nucleic Acids Res 1994, 22:4673-4680.

26. Tamura K, Peterson D, Peterson N, Stecher G, Nei M, Kumar S: MEGA5: molecular evolutionary genetics analysis using maximum likelihood, evolutionary distance, and maximum parsimony methods. Mol Biol Evol 2011, 28:2731-2739

27. Darriba D, Taboada GL, Doallo R, Posada D: jModelTest 2: more models, new heuristics and parallel computing. Nat Methods 2012, 9(8):772.

28. Glukhov AS, Krutilina Al, Shlyapnikov MG, Severinov K, Lavysh D, Kochetkov W, McGrath JW, De Leeuwe C, Shaburova OV, Krylov VN, Akulenko NV, La K: Genomic analysis of Pseudomonas putida phage tf with localized singlestrand DNA interruptions. PloS one 2012, 7:e51163.

29. Wang I, Smith DL, Young R: HOLINS: The Protein Clocks of Bacteriophage Infections. Annu Rev Microbiol 2000, 54:799-825.

30. Hertveldt K, Lavigne R, Pleteneva E, Sernova N, Kurochkina L, Korchevskii R, Robben J, Mesyanzhinov V, Krylov VN, Volckaert G: Genome comparison of Pseudomonas aeruginosa large phages. J Mol Biol 2005, 354:536-545.

31. Feiss M, Rao VB: The bacteriophage DNA packaging machine. Advances in experimental medicine and biology 2012, 726:489-509.

32. Shen $X$, Li M, Zeng Y, Hu X: Functional identification of the DNA packaging terminase from Pseudomonas aeruginosa phage PaP3. Arch Virol 2012, 157(11):2133-2141.

33. Segalés J, Olvera A, Grau-Roma L, Charreyre C, Nauwynck H, Larsen L, Dupont K, McCullough K, Ellis J, Krakowka S, Mankertz A, Fredholm M, Fossum C, Timmusk S, Stockhofe-Zurwieden N, Beattie V, Armstrong D, Grassland B, Baekbo P, Allan G: PCV-2 genotype definition and nomenclature. Vet Rec 2008, 162:867-868

34. Fauquet CM, Stanley J: Revising the way we conceive and name viruses below the species level: a review of geminivirus taxonomy calls for new standardized isolate descriptors. Arch Virol 2005, 150:2151-2179.

doi:10.1186/1471-2164-15-7

Cite this article as: Eller et al:: UFV-P2 as a member of the Luz24likevirus genus: a new overview on comparative functional genome analyses of the LUZ24-like phages. BMC Genomics 2014 15:7. 